\section{SOI: 1.1 TAA $\quad$ DOI: $10.15863 /$ TAS}

\section{International Scientific Journal}

\section{Theoretical \& Applied Science}

p-ISSN: 2308-4944 (print) e-ISSN: 2409-0085 (online)

Year: $2016 \quad$ Issue: 2 Volume: 34

Published: 29.02.2016 http://T-Science.org

SECTION 31. Economic research, finance, innovation, risk management.
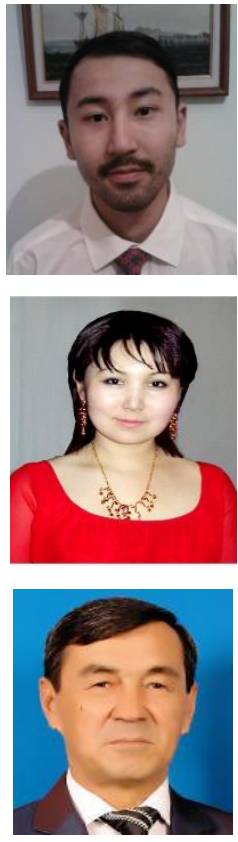

Ersultan Zhomartuly Shalkar undergraduate,

Kazakhstan Engineering and Pedagogical University of Peoples Friendship, Kazakhstan yernar_shalkharov@bk.ru

Aygul Kuanyshevna Kupesheva candidate of economic Sciences, Senior lecturer Head of the Department "Economy", Kazakhstan Engineering and Pedagogical University of Peoples Friendship, Kazakhstan kup_aigul@mail.ru

Borash Smailovich Myrzaliev doctor of economic Sciences, Professor, head of Department of Economics H.A.Yassawe International Kazakh-Turkish University, Kazakhstan

\title{
OPTIMIZATION OF THE ECONOMIC INDICATORS BUILDING OBJECT IN THE PREPARATION OF THE CALENDAR SCHEDULE OF CONSTRUCTION RISK ACCORDING TO SIMPLIFIED FORMULA OF MONTE CARLO METHOD
}

Abstract: This article is dedicated to economically sound phenomenon, the best known and characterized as risks during construction that are of particular relevance through impact on scheduling, as well as podpravka timetable data risk according to the simplified formula in accordance with the Monte Carlo method, which, in turn, has an impact on the estimated cost of construction as a whole.

Key words: schedule, weight importance, crisis, innovation, investment, risk, range, efficiency analysis, estimated cost, optimization.

Language: Russian

Citation: Shalkar EZ, Kupesheva AK, Myrzaliev BS (2016) OPTIMIZATION OF THE ECONOMIC INDICATORS BUILDING OBJECT IN THE PREPARATION OF THE CALENDAR SCHEDULE OF CONSTRUCTION RISK ACCORDING TO SIMPLIFIED FORMULA OF MONTE CARLO METHOD. ISJ Theoretical \& Applied Science, 02 (34): 21-28.

Soi: http://s-o-i.org/1.1/TAS-02-34-3 Doi: crossef http://dx.doi.org/10.15863/TAS.2016.02.34.3

ОПТИМИЗАЦИЯ ЭКОНОМИЧЕСКОГО ПОКАЗАТЕЛЯ СТРОИТЕЛЬНОГО ОБЬЕКТА ПРИ СОСТАВЛЕНИИ КАЛЕНДАРНОГО ГРАФИКА СТРОИТЕЛЬНЫХ РАБОТ С УЧЕТОМ РИСКОВ ПО УПРОЩЕННОЙ ФОРМУЛЕ МЕТОДА МОНТЕ-КАРЛО

Аннотация: Данная статья посвящается экономически обоснованному феномену, наиболее известным и характеризуемым как риски при строительстве, которые имеют особую актуальность посредством влияния на составление календарного графика, а также подправке календарного графика с учетом данных рисков по упрощенной формуле в соответствие с методом Монте-Карло, который, в свою очередь, имеет влияние на сметную стоимость строительства в целом.

Ключевые слова: календарный график, вес значимости, кризис, инновации, инвестиции, риски, диапазон, анализ эффективности, сметная стоимость, оптимизация.

В наши дни строительный сектор является одним из наиболее развитых направлений в бизнес среде любого государства. Актуальность таковой популярности обоснована повышенной необходимостью в реализации строительной политики Казахстана на макроэкономическом уровне [1]. Помимо прочего, спрос на объекты эксплуатации растет в арифметической 
прогрессии независимо от вида строительства. Тем не менее, риски являются неотъемлемый частью экономической науки. Даже в процессе строительства имеется ряд положений, относящихся к рисковым ситуациям [2]. На сегодняшний день менеджмент календарного графика обретает все большую значимость в строительном секторе [3]. Соответственно, при грамотном инженерном расчете календарного графика в процессе строительства необходимо учитывать специфическую информацию касательно оптимизации строительного процесса как с экономической позиции, так и с инженерной точки зрения [4]. Для этого необходимо, прежде всего, провести S.W.O.T анализ необходимости доработки метода Монте-Карло с целью определить эффективность разрабатываемого метода в прикладном производстве.

\begin{tabular}{|c|c|}
\hline $\mathbf{S}$ & W \\
\hline $\begin{array}{c}\text { Stretch } \\
\text { (сильные стороны) }\end{array}$ & $\begin{array}{l}\text { Weaknesses } \\
\text { (слабые стороны) }\end{array}$ \\
\hline 1.Качество строительных конструкций объекта. & $\begin{array}{l}\text { 1.Затрата большего количества времени, } \\
\text { нежели без учета времени на риски при } \\
\text { строительстве объекта. }\end{array}$ \\
\hline 2.Качество строительных материалов. & $\begin{array}{ccc}\begin{array}{c}\text { 2.Увеличение } \\
\text { строительного объекта. }\end{array} & \text { сметной } & \text { стоимости } \\
\end{array}$ \\
\hline $\begin{array}{ccc}\text { 3.Систематический } & \text { подход } \\
\text { строителей к выявлению рисков при строительстве. }\end{array}$ & $\begin{array}{l}\text { 3.Коэффициент погрешности при составления } \\
\text { календарного графика с учетом рисков до } 3 \% \text {. }\end{array}$ \\
\hline $\begin{array}{l}\text { 4. Экономический выгодная позиция составления } \\
\text { календарного графика с учетом рисков. }\end{array}$ & 4.Линейность календарного планирования. \\
\hline $\mathbf{O}$ & $\mathbf{T}$ \\
\hline $\begin{array}{c}\text { Opportunities } \\
\text { (возможности) }\end{array}$ & $\begin{array}{l}\text { Threats } \\
\text { (риски) }\end{array}$ \\
\hline $\begin{array}{l}\text { 1.Дальнейший учет рисков при составлении } \\
\text { календарного графика строительных работ. }\end{array}$ & $\begin{array}{ccc}\text { 1. Непредвиденные } & \text { обстоятельства } & \text { в } \\
\text { строительных процессах. } & & \end{array}$ \\
\hline $\begin{array}{l}\text { 2.Учет неопределенностей в строительстве, то } \\
\text { есть веса значимости объекта. }\end{array}$ & \\
\hline $\begin{array}{l}\text { 3.Определения сметной стоимости объекта в } \\
\text { зависимости от рисков при строительстве. }\end{array}$ & \\
\hline $\begin{array}{c}\text { 4.Изучение степени влияния рисков } \\
\text { строительстве на макроэкономическом уровне. }\end{array}$ & \\
\hline
\end{tabular}

В соответствие с выше приведенным анализом можно утвердить необходимость в модернизации стандартной формулы расчета календарного графика в процессе строительства по методу Монте-Карло по более упрощенной формуле для оптимизации ресурсов и времени в строительстве. Для того чтобы определить экономический показатель в строительстве, которым является сметная стоимость строительства, необходимо найти продолжительность времени строительства, а для этого следует рассмотреть календарный график с учетом рисков при строительстве.

Существенное влияние на сроки и качество выполнения инвестиционно-строительных проектов оказывают неблагоприятные ситуации и их последствия, которые интерпретируются как риски [5]. Ни один из известных производственных процессов не подвержен такому большому количеству различных опасностей, которые необходимо своевременно выявлять, оценивать в их совокупности и анализировать на стадии подготовки проекта в последующих этапах строительства и эксплуатации [6]. В связи с увеличением количества факторов риска, методики управления ими должны постоянно совершенствоваться. Все участники проекта (инвесторы, заказчики, подрядчики, органы государственной власти, общественные организации и т.д.) расширяют диапазон причин возникновения рисков. Кроме этого, персонал, отвечающий за управление рисками, должен использовать не интуитивный подход, а систематический, благодаря которому все риски будут выявлены и своевременно взяты под контроль, что приведет к улучшению качества строительного процесса [7]. Все это свидетельствует о том, что управление рисками следует рассматривать не как единовременное действие, а как группу целенаправленных действий, образующих единый механизм управления рисками, интегрированный в процесс управления инвестиционно-строительными проектами. Так же, не маловажным фактором является и вес значимости объекта, или ИСП. Проблема риска по реализации ИСП в экономике РК достаточно актуальна, так как в настоящее время литературные источники, посвященные 


\begin{tabular}{|c|c|c|c|c|c|c|}
\hline Impact Factor: & $\begin{array}{l}\text { ISRA (India) } \\
\text { ISI (Dubai, UAE } \\
\text { GIF (Australia) } \\
\text { JIF }\end{array}$ & $\begin{array}{r}=1.344 \\
=0.829 \\
=0.564 \\
=1.500\end{array}$ & $\begin{array}{l}\text { SIS (USA) } \\
\text { PИHЦ (Russia) } \\
\text { ESJI (KZ) } \\
\text { SJIF (Morocco) }\end{array}$ & $\begin{array}{l}=0.912 \\
=0.179 \\
=1.042 \\
=\mathbf{2 . 0 3 1}\end{array}$ & $\begin{array}{l}\text { ICV (Poland) } \\
\text { PIF (India) }\end{array}$ & $\begin{array}{l}=6.630 \\
=1.940\end{array}$ \\
\hline
\end{tabular}

таковым рискам в этой области отсутствуют, фундаментальные исследования, по существу, не проводились [8]. Объектом исследования является риски и вес значимости при строительстве. Как справедливо отметила в своем исследовании магистр технических наук Кинаят Л.А настоящие риски более целесообразно анализировать по методу Монте-Карло [9].

Построение алгоритмической схемы для вывода упрощенной формулы подтверждающий методику Монте-Карло наглядно продемонстрирован в ниже приведенной схеме. (см.рис. 1)

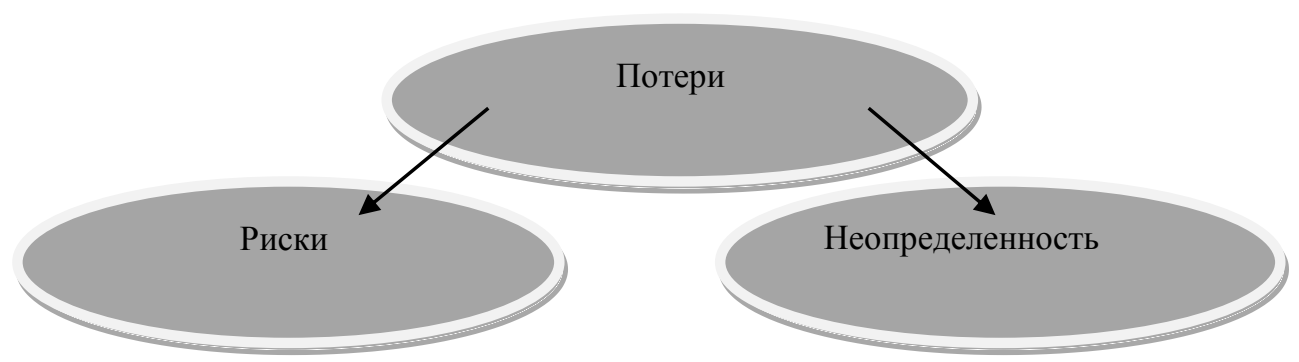

Рисунок 1 - Структурный анализ потерь дней в зависимости от риска и неопределенности.

Так же в соответствующих исследованиях. была уставлена взаимосвязь между риском, потерей и неопределенностью [9]. Но вопрос обстоит в другом. В потерях, так как из-за рисков при строительстве мы теряем количество дней, соответственно для составления календарного графика мы должны найти количество дней с учетом рисков при строительстве. Заключительным положением остается интерпретация термина "неопределенность". Неопределенность - это неполнота или неточность информации об условиях реализации проекта, то есть вес значимости проекта. Риски классифицируются на внешние, организационные и технические (см.схему 1 ).

Анализ рисков на стадии строительства (см.рис. 2)

\section{Схема 1 - Классификация рисков по сфере их проявления.}

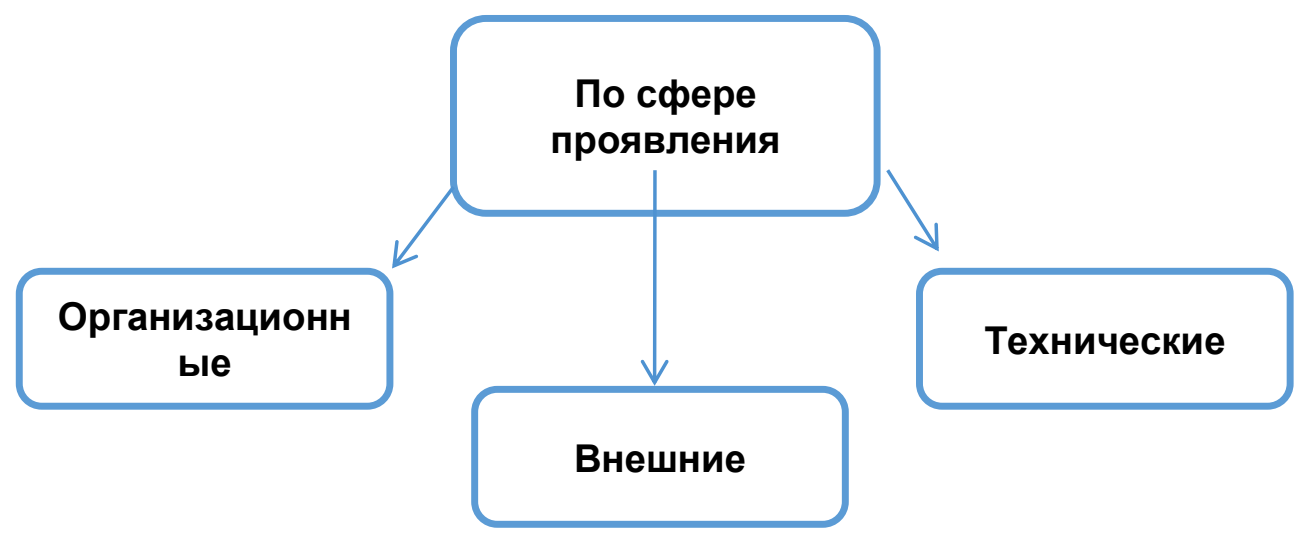




\begin{tabular}{|c|c|c|c|c|c|c|}
\hline Impact Factor: & $\begin{array}{l}\text { ISRA (India) } \\
\text { ISI (Dubai, UAE } \\
\text { GIF (Australia) } \\
\text { JIF }\end{array}$ & $\begin{array}{l}=1.344 \\
=0.829 \\
=0.564 \\
=1.500\end{array}$ & $\begin{array}{l}\text { SIS (USA) } \\
\text { PИНЦ (Russia) } \\
\text { ESJI (KZ) } \\
\text { SJIF (Morocco) }\end{array}$ & $\begin{array}{l}=0.912 \\
=0.179 \\
=1.042 \\
=2.031\end{array}$ & $\begin{array}{l}\text { ICV (Poland) } \\
\text { PIF (India) }\end{array}$ & $\begin{array}{l}=6.630 \\
=1.940\end{array}$ \\
\hline
\end{tabular}

\section{Анализ рисков на стадии строительства}

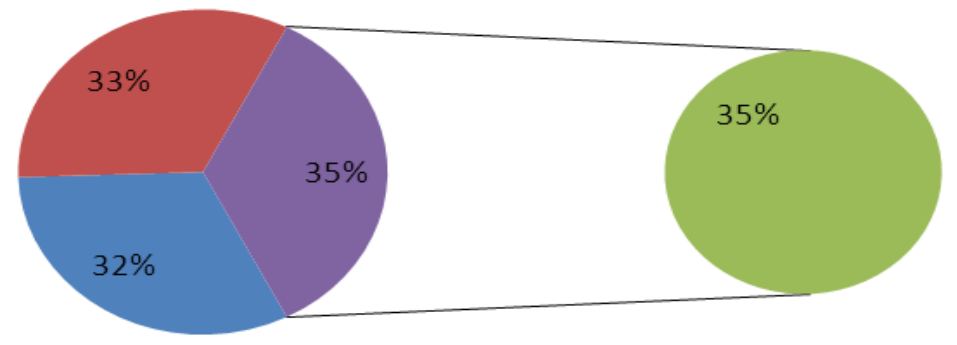

— организационные риски

внешние риски

шехнические риски

\section{Рисунок 2 - Анализ рисков на стадии строительства.}

Вес значимости характеризуется своим коэффициентом.

$$
\mathrm{V}_{\mathrm{i}}=\frac{\mathrm{x}_{\mathrm{ci}}}{\mathrm{n}^{2}},
$$

где $\mathrm{x}$ - сумма степени важности задачи, Vкоэффициент веса значимости, n-количество задач.

Данные для расчета взяты из вышеуказанных исследований Кинаят Л.А. (см.рис. 3).

Таблица 1

\section{Пример матрицы предпочтений.}

\begin{tabular}{|c|c|c|c|c|c|c|c|c|c|c|c|c|c|c|c|}
\hline $\mathbf{K i}$ & K1 & $\mathbf{K 2}$ & $\mathbf{K} \mathbf{3}$ & K4 & $\mathbf{K 5}$ & K6 & K7 & $\mathbf{K 8}$ & Ke9 & K10 & K11 & K12 & $\sum_{1}$ & $\begin{array}{c}\text { Ранг } \\
\text { качества }\end{array}$ & $\begin{array}{c}\text { Bсе } \\
\text { значимос } \\
\text { Ти }\end{array}$ \\
\hline $\mathbf{K} 1$ & 1 & 2 & 1 & 2 & o & 2 & 2 & 2 & 1 & o & $\mathrm{O}$ & 1 & 14 & 5 & 0,09722 \\
\hline $\mathrm{K} 2$ & o & 1 & 2 & 2 & 1 & 2 & 2 & 2 & 1 & 1 & 1 & 1 & 16 & 3 & 0,11111 \\
\hline $\mathbf{K} 3$ & 1 & $\mathrm{O}$ & 1 & 2 & $\mathrm{O}$ & 2 & 2 & 1 & 0 & 1 & 1 & 0 & 11 & 7 & 0,07639 \\
\hline $\mathbf{K} 4$ & 0 & $\mathrm{O}$ & $\mathrm{O}$ & 1 & 1 & 1 & 2 & 2 & 1 & $\mathbf{1}$ & $\mathrm{O}$ & 0 & 9 & 8 & 0,0625 \\
\hline $\mathbf{K 5}$ & 2 & 1 & 2 & 1 & 1 & 1 & 2 & 2 & 2 & 1 & $\mathrm{O}$ & 0 & 15 & 4 & 0,10417 \\
\hline K6 & 0 & $\mathrm{O}$ & $\mathrm{O}$ & 1 & 1 & 1 & 2 & 2 & 0 & o & $\mathrm{O}$ & 0 & 7 & 9 & 0,04861 \\
\hline $\mathbf{K} 7$ & $\mathrm{O}$ & $\mathrm{O}$ & $\mathrm{O}$ & O & o & $\mathrm{O}$ & 1 & 1 & 1 & o & $\mathrm{O}$ & 0 & 3 & 10 & 0,02083 \\
\hline K88 & 0 & $\mathrm{O}$ & 1 & o & $\mathrm{O}$ & 0 & 1 & 1 & 0 & 0 & $\mathrm{O}$ & 0 & 3 & 10 & 0,02083 \\
\hline Ke & 1 & 1 & 2 & 1 & $\mathrm{O}$ & 2 & 1 & 2 & 1 & 0 & $\mathrm{O}$ & 0 & 12 & 6 & 0,08333 \\
\hline K10 & 2 & 1 & 1 & 1 & 1 & 2 & 2 & 2 & 2 & 1 & 1 & 0 & 18 & 2 & 0,125 \\
\hline K11 & 2 & 1 & 1 & 2 & 2 & 2 & 2 & 2 & 2 & 1 & 1 & 1 & 19 & 1 & 0,13194 \\
\hline $\mathbf{K} 12$ & 1 & 1 & 2 & 2 & 2 & 2 & 2 & 2 & 2 & 2 & 1 & 1 & 18 & 2 & 0,125 \\
\hline
\end{tabular}

Пример расчета коэффициента веса значимости V1 $=14 / 144=0,09722$

Где:

$\square$ К1-Некорректная постановка задачи Заказчиком

$\square$ К2-Задержка получения исходных данных

$\square$ К3-Задержка выпуска документации проектировщиками $\square$ К4-Низкий уровень организации строительного процесса, логистики и расчетов в строительстве

$\square$ К5-Начало работ до разработки проектной документации

$\square$ К6-Рост цен на строительные материалы, изделия и конструкции

$\square$ К7-Несовершенное законодательство 
$\square$ Изменение законодательства, вступление в силу новых технических регламентов, постановлений, приказов правительства, касающиеся данного проектирования

$\square$ К8-Задержка согласований в

уполномоченных ведомствах и комитетах

$\square$ К9-Высокие непроизводственные потери застройщиков на этапе подготовки строительства и сдачи в эксплуатацию, коррупция данных

$\square$ К10-Принятие неточных исходных

$\square$ К11-Ошибки при проектировании (например, принятие некорректных технических решений, потеря взаимосвязи между разделами проекта и т.п.)

$\square$ К12-Внесение глобальных изменений в проект Заказчиком в процессе проектирования (например, смена концепции объекта, изменение конфигурации объекта, внесение изменений в конструкции, обеспечивающие надежность и безопасность объекта и т.п.)

Для упрощенной формулы мы не учитываем V1 - V2 коэффициент веса значимости, так как условно берем что, это не относится к строительству. Следовательно, по гипотезе формула выглядит следующим образом

$$
\mathrm{S}=\left(\bar{\sum} V+P_{T}\right) \times \mathrm{T}
$$

Где S- количество дней по календарному графику

$\sum \mathrm{V}$ - среднее значение коэффициент веса значимости

PT- вероятность проявления технических рисков

T - количество дней по календарному графику без учета рисков

Тогда подставляя исходные данные из диссертационной работы Кинаят Л.А. получаем следующее значение в соответствие, с которым:

$$
\begin{aligned}
& \bar{\Sigma} \quad V=0.868543 \\
& P_{T}=0.35 \\
& T=302 \\
& \mathrm{~S}=\left(\begin{array}{ll}
\frac{\Sigma}{\Sigma} & K+P_{T}
\end{array}\right) \times \mathrm{T}= \\
& (0.868543 \quad+0.35) \times \\
& 302=367.99998 \approx 368
\end{aligned}
$$

Соответственно, по методу Монте-Карло в процессе строительства общественного объекта количество дней в календарном графике составляет 368 дней, что, следовательно, значительно упрощает общую методологию расчета календарного графика с учетом рисков в строительстве [10].

Степень влияния на сметную стоимость количество дней, выделенных на подправку календарного графика повышается по критерию "время -ресурсы -стоимость ", что в свою очередь дает возможность избегать иные риски при строительстве [11]. К рискам в строительстве относятся не только строительные процессы, но и неблагоприятные условия в процессе строительства и заполнение надлежащих документов (ежедневный отчет, ежемесячный отчет, договора между подрядчиками, поставщиками, утверждение корректировок в действующий проект и т.д.). Все это приводит к торможению строительного процесса и отражается дополнительными днями в календарном графике [12]. Качество выполненных работ в строительстве прямо зависит от времени и соблюдения технологии. Исходя из вышеуказанного следует знать, что для строительства можно выбрать наиболее оптимальный диапазон времени, который, иными словами, находит точку соприкосновения с количеством дней и качеством строительства, а также сметной стоимостью от продолжительности времени строительства с целью определения экономической оптимальной стоимости проекта строительства [13].

Для начала надо разделить время в процентном соотношении чтобы понять сколько времени уходит на строительные процессы: земляные работы основных несущих конструкции, ограждающих конструкций, внешней отделки, и внутренней отделки. Основную несущую и ненесущую конструкции необходимо объединить, так как здания, сооружения бывают с конструкциями, несущими и в тоже время ограждающими [14]. В соответствующем расчете необходимо взять время, затраченное на каждый из строительных процессов и разделить на окончательное время завершения строительства. (см.рис. 4) 


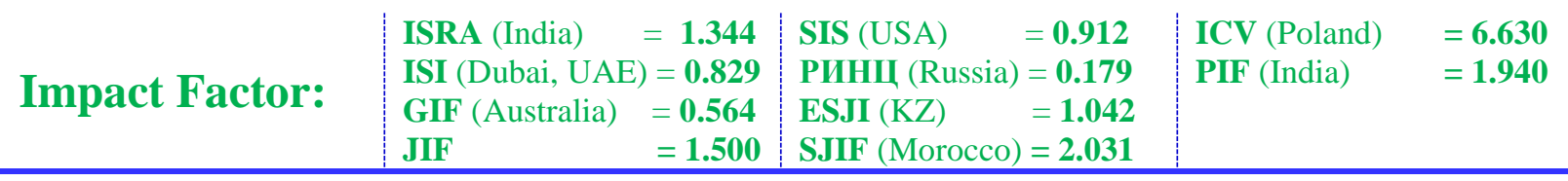

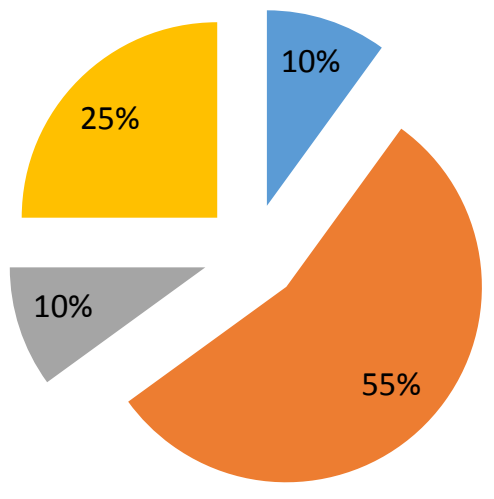

—емляные работы

- Несущая конструкция и ограждающая конструкция (ненесущая)

Внешняя отделка

— Внутрення отделка

Рисунок 4 - Пример основных работ в строительном процессе.

Качество строительных конструкций и материалов зависит от времени использования, хранения и условия хранений материалов, так как неблагоприятные условия для материалов и конструкций понижают их первоначальные физические и химические характеристики, тем самым влияя на качество самого строительного объекта. (см.рис. 5)

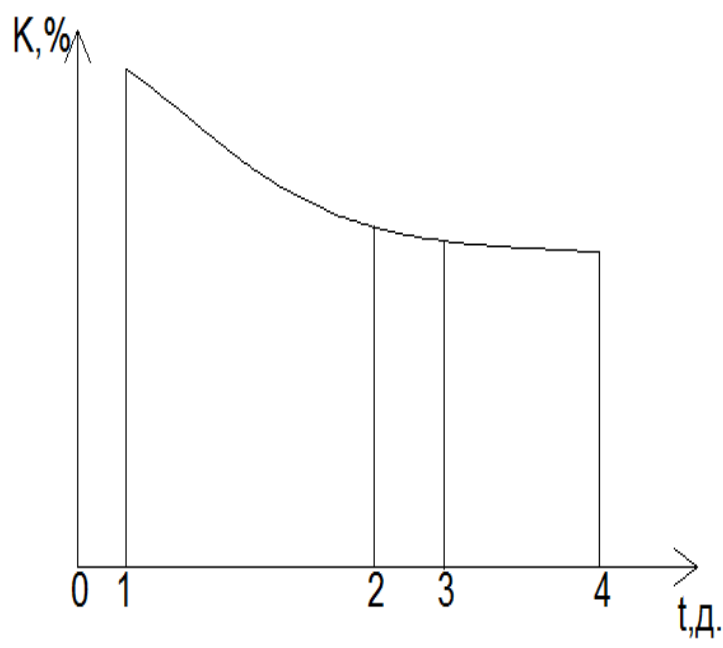

Рисунок 5 - График зависимости качества от продолжительности .

где: К- качество строительных конструкций, в процентах.,

0-1 промежуток времени земляных работ.,

1-2 Строительные процессы несущих и ограждающих конструкций.,

2-3 Строительные процессы внешней отделки., 3-4 Строительные процессы внутренней отделки.
Аналогичный график можно наблюдать с позиции бюджетного управления, в соответствие с которым стоимость строительных процессов находится в зависимости от промежуточного отрезка времени объекта строительства. (см.рис.6) 


\begin{tabular}{l|lrl|l|ll} 
& ISRA (India) & $=\mathbf{1 . 3 4 4}$ & SIS (USA) & $=\mathbf{0 . 9 1 2}$ & ICV (Poland) & $=\mathbf{6 . 6 3 0}$ \\
Impact Factor: & ISI (Dubai, UAE) $=\mathbf{0 . 8 2 9}$ & PUHL (Russia) $=\mathbf{0 . 1 7 9}$ & PIF (India) & $=\mathbf{1 . 9 4 0}$ \\
& GIF (Australia) & $\mathbf{0 . 5 6 4}$ & ESJI (KZ) & $=\mathbf{1 . 0 4 2}$ & & \\
& JIF & $\mathbf{1 . 5 0 0}$ & SJIF (Morocco) $=\mathbf{2 . 0 3 1}$ & & \\
\hline
\end{tabular}

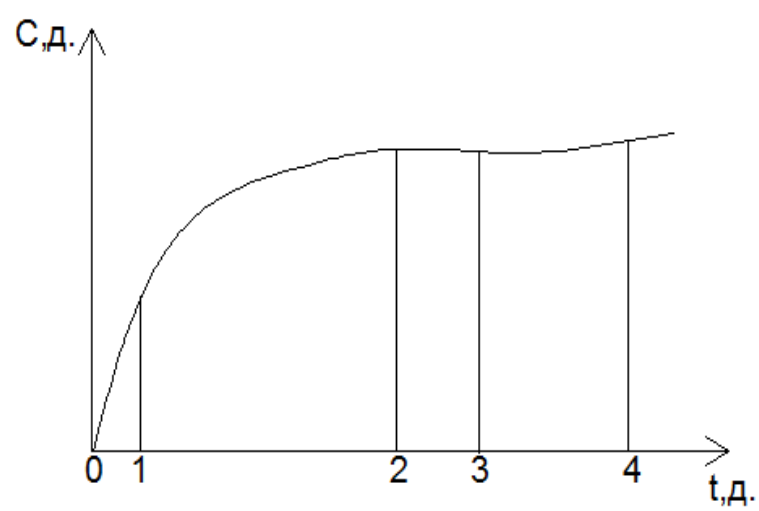

\section{Рисунок 6 - График зависимости сметной стоимости строительных процессов от времени.}

где: С- сметная стоимость строительных процессов, в деньгах.0-1 промежуток времени земляных работ.,

0-1 промежуток времени земляных работ.,

1-2 Строительные процессы несущих и ограждающих конструкций.,

2-3 Строительные процессы внешней отделки.,
3-4 Строительные процессы внутренней отделки.

При накладывании двух графиков можно увидеть степень влияния времени на качество и на сметную стоимость отдельных строительных процессов. (см.рис.7)

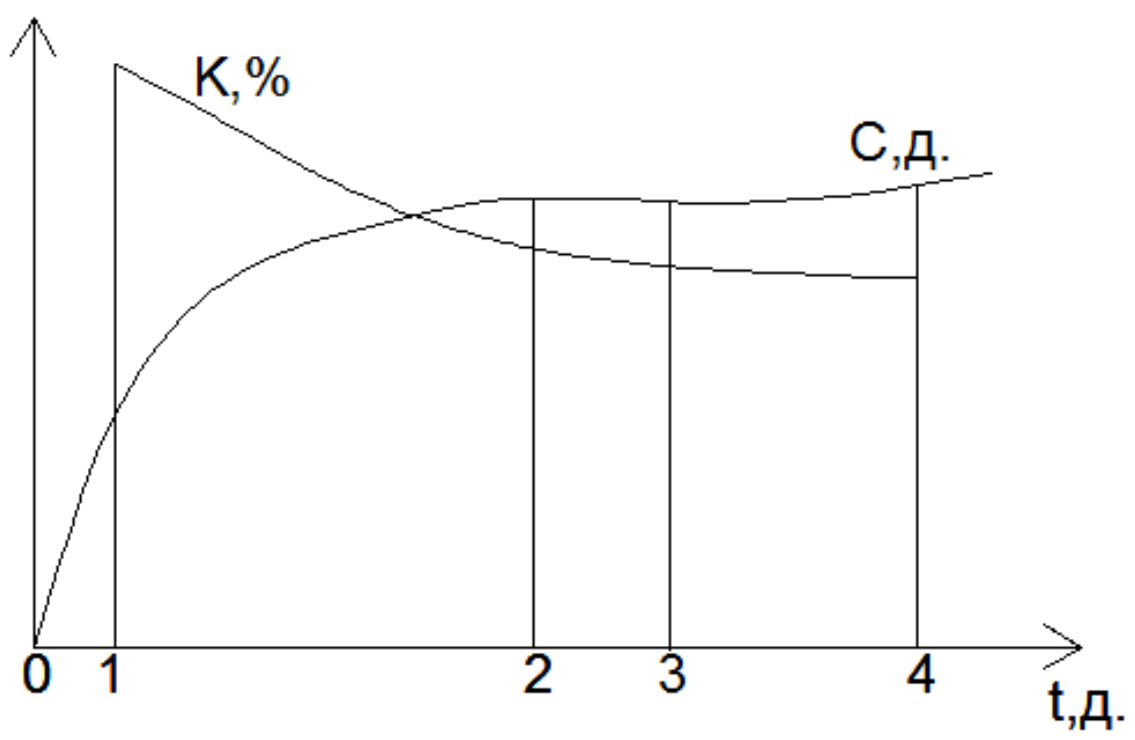

Рисунок 7- График зависимости качества и сметной стоимости строительных процессов от времени.

где: К - качество строительных конструкций, в процентах., С- сметная стоимость строительных процессов, в деньгах.

0-1 промежуток времени земляных работ.,

1-2 Строительные процессы несущих и ограждающих конструкций.,

2-3 Строительные процессы внешней отделки.,
3-4 Строительные процессы внутренней отделки.

Таким образом можно подытожить цели настоящей статьи: экономический показатель был оптимизирован тем, что график зависимости качества и сметной стоимости строительных процессов от продолжительности времени строительства объекта показал, что увеличение 


\begin{tabular}{|c|c|c|c|c|c|c|}
\hline Impact Factor: & $\begin{array}{l}\text { ISRA (India) } \\
\text { ISI (Dubai, UAE } \\
\text { GIF (Australia) } \\
\text { JIF }\end{array}$ & $\begin{array}{l}=1.344 \\
=0.829 \\
=0.564 \\
=1.500\end{array}$ & $\begin{array}{l}\text { SIS (USA) } \\
\text { PИНЦ (Russia } \\
\text { ESJI (KZ) } \\
\text { SJIF (Morocco }\end{array}$ & $\begin{array}{l}=0.912 \\
=0.179 \\
=1.042 \\
=2.031\end{array}$ & $\begin{array}{l}\text { ICV (Poland) } \\
\text { PIF (India) }\end{array}$ & $\begin{array}{l}=6.630 \\
=1.940\end{array}$ \\
\hline
\end{tabular}

сметной стоимости при строительстве с учетом рисков приводит к уменьшение сметной стоимости нежели при не выявления рисков при строительстве

\section{References:}

1. Kennedy O. Aduda, WimZeiler, GertBoxem, Kevin de Bont (2015) Necessity of entering innovation in building policy modernization in Central Asia, espessially in Kazakhstan.Original Research Article.Procedia Building Economy Science, Volume. 32, 2015, Pages 723-730.

2. Peter S. Cornish, AvijitChoudhury, Ashok Kumar, Sudipta Das, KuntalikaKumbakhar, Shane Norrish, Shivendra Kumar (2015) Risk issues in building economics, especially in time management and project management cases.Original Research Article.Agricultural Systems, Volume 137, July 2015, Pages 180190.

3. Denis J.B Shaw (2015) Actual issues of project management and time management in state building policy and modern macroeconomic policy. Economical Policy, Volume 18, Issue 8, November. 2015, Pages 991-1015.

4. Brian Graves, Alain Wong, KianMousavi, Christina Canter, Amit Kumar (2015) Multidisciplinary issues of modernization economical building policy by special engineering methodology: comparative approach. Original Research Article.Annals of Economical Engineering and Building, Volume 85, November 2015, Pages 481-487.

5. Sarah Foster, Matthew Knuiman, Lisa Wood, Billie Giles-Corti (2015) Validity degree of building risks in time calculation process. Original Research Article. Journal of Legal Building Science, Volume 36, December 2015, Pages 112-117.

6. Geert Bekaert, Campbell R. Harvey, Christian T. Lundblad, Stephan Siegel (2015) Pressing questions of some dangers in an error a building process. Original Research Article. Journal of Corporate Finance in Building, In Press, Accepted Manuscript, Available online 31 December 2015.
7. Ryan Sullivan, Allan Timmermann, Halbert White (2015) Intuitive and systematic issue in building time management process. Original Research Article. Journal of Econometrics, Volume 105, Issue 1, November 2015, Pages 249-286.

8. Tamas Toth, Zoltan Sebestyen (2015) Timevarying Risks of Construction Projects Original Research Article Procedia Engineering, Volume 123, 2015, Pages 565-573.

9. (2013) Master's thesis Kinat L. A. "Risks in scheduling the construction of Monte Carlo" 2013. 72 p.

10. Ann Villow. Michael N.Merdock, Anshy Morrell, Debra Quasias (2015) Special building methodology including Monte-Carlo using analogy in building risks. Original Research Article. Building Science Systems, Volume 115, September 2015, Pages 39-48.

11. Ocean O.Right. Michael Sheel (2015) Estimate cost problem of building in economical issues. Original Article. Modern Economy, Volume 56, Issue 86, June. 2015, Pages 516-523.

12. Aamir Hurash, Salena Burgh, Sherts Murreran (2015) Additional reasoning in building economy. Original Research Article. Economical Engineering and Building, Volume 113, March 2015, Pages 481-487.

13. Elen G.Konrad, Silvia Cornerfell, Blanca Tillares (2015) Some scientific issues in time management of building optimization process. Original Research Article. Modern Microeconomy, Volume 13, December 2015, Pages 96-125.

14. Luisa O.Dennel, Den M.Conergan, Lailo Tull (2015) Types of constructions and its economical value. Original Research Article. Modern Microeconomy, Volume 18, April 2015, Pages 56-74. 